\title{
Validación de CitrusVol para el ajuste de volúmenes de caldo en tratamientos contra araña roja en cítricos
}

\author{
A. Fonte ${ }^{1}$, C. Garcerá ${ }^{1}$, E. Moltó ${ }^{1}$, A. Tena ${ }^{2}$ y P. Chueca ${ }^{1}$ \\ 1 Centro de Agroingeniería, Instituto Valenciano de Investigaciones Agrarias (IVIA); chueca_pat@gva.es \\ 2 Centro de Protección Vegetal y Biotecnología, Instituto Valenciano de Investigaciones Agrarias (IVIA)
}

\begin{abstract}
Resumen: Para optimizar la aplicación de fitosanitarios, el IVIA ha desarrollado una herramienta de recomendación de volumen de caldo para las aplicaciones mediante turboatomizadores en cítricos: CitrusVol. Esta herramienta permite ajustar el volumen de caldo a las características del cultivo, al tipo de plaga o enfermedad y al tipo de producto fitosanitario. El objetivo de este trabajo fue validar CitrusVol para el control de la araña roja, Tetranychus urticae Koch, en clementinos, dado que es una de las principales plagas del cultivo. Para ello, se compararon los tratamientos fitosanitarios siguiendo las recomendaciones de CitrusVol con los tratamientos realizados de manera convencional por la explotación. Se evaluó, por un lado, la distribución del caldo en la copa estimando el porcentaje de recubrimiento. Por otro lado, se determinó la eficacia de los tratamientos, para lo que se realizaron muestreos previos y posteriores a los tratamientos fitosanitarios y se determinó el porcentaje de hojas sintomáticas ocupadas por araña. Los resultados mostraron que con el empleo de CitrusVol se consiguió una reducción media del volumen de caldo de 33,5\%, lo que implicó una reducción del recubrimiento con el volumen ajustado respecto al convencional. Sin embargo, no se encontraron diferencias significativas en el porcentaje de hojas sintomáticas ocupadas por araña roja en función del volumen de caldo aplicado. Esto permite concluir que la eficacia fue la misma y que el volumen recomendado por CitrusVol es adecuado para el control de esta plaga en clementinos.
\end{abstract}

Palabras clave: Tetranychus urticae, clementino, recubrimiento, turboatomizador

\section{Introducción}

A pesar de los notables avances en el control biológico de plagas y enfermedades, la aplicación de productos fitosanitarios (PPP) sigue siendo el método más común para proteger los cítricos. Los equipos habitualmente empleados en estas aplicaciones son pulverizadores hidráulicos asistidos por aire, comúnmente conocidos como turboatomizadores. En estos equipos más del $50 \%$ del caldo pulverizado se puede perder al suelo o la atmósfera como resultado de la deriva, evaporación, escorrentía y/o lavado de los productos [1].

Con el fin de mejorar la eficiencia de estas aplicaciones, se está trabajando en la racionalización de las mismas ajustando adecuadamente la cantidad de producto según las necesidades reales y las condiciones específicas de la aplicación (vegetación a cubrir, plaga a controlar, pesticidas usados y maquinaria). De esta manera se trata de evitar la práctica común de hoy en día de aplicar grandes cantidades de producto para garantizar resultados, sin tener en cuenta que esto normalmente conlleva una liberación excesiva de productos que permanecen en los alimentos y contaminan el medio ambiente, y que también aumenta los costes. En este 


\section{CONGRESO IBÉRICO DE AGROINGENIERÍA \\ X CONGRESSO IBÉRICO DE AGROENGENHARIA \\ 3 - 6 septiembre 2019, Huesca - España}

sentido el IVIA ha desarrollado para cítricos "CitrusVol", una herramienta informática de soporte de decisión al técnico o agricultor para calcular el volumen de caldo de la aplicación con turboatomizador ajustado a las características de la vegetación objetivo (volumen de copa, marco de plantación, densidad foliar y nivel de poda), al tipo de plaga o enfermedad, y al tipo de producto fitosanitario [2]. Esta herramienta se basa en datos científicos obtenidos anteriormente con respecto al depósito mínimo requerido para lograr la máxima eficacia, la eficiencia de la aplicación con turboatomizadores en parcelas de cítricos y la caracterización del cultivo [1,3-6]. Esta herramienta está disponible gratuitamente en la página web de Gestión Integrada de Plagas de cítricos del IVIA (http://gipcitricos.ivia.es/) y en la aplicación para iOS y Android "Gipcitricos IVIA".

La araña roja, Tetranychus urticae Koch (Acari: Tetranychidae), es el ácaro que más impacto económico tiene en los cultivos citrícolas de España, siendo los clementinos particularmente sensibles a su ataque. Al alimentarse del contenido de las células epidérmicas de los tejidos vegetales, ocasiona manchas cloróticas en las hojas y decoloraciones y cicatrices en los frutos, depreciándolos comercialmente. Si el ataque es fuerte, puede provocar un debilitamiento generalizado del árbol ocasionando una merma en su producción y crecimiento.

El objetivo de este trabajo fue validar la herramienta CitrusVol para los tratamientos contra araña roja en clementinos. Para ello se comparó la eficacia de las aplicaciones basadas en CitrusVol con las aplicaciones realizadas de forma habitual por los productores.

\section{Materiales y métodos}

\subsection{Diseño experimental}

El experimento se llevó a cabo en una parcela comercial de mandarinos de la variedad “Clemenules" ubicada en Chiva (Valencia; 39 26 32"N, $0^{\circ} 33^{\prime 2} 23^{\prime \prime} \mathrm{O}$ ). La parcela tenía un marco de plantación de $6 \times 3 \mathrm{~m}$, con las filas dispuestas en orientación N-S.

La parcela se dividió en dos bloques de tamaño similar, en uno se realizaron los tratamientos contra araña roja con el volumen de caldo recomendado por CitrusVol (denominado Volumen ajustado, $\mathrm{V}_{\mathrm{A}}$ ), y en otro con el volumen empleado habitualmente por los técnicos de la explotación agrícola (Volumen convencional, Vc), durante 2 campañas: 2016 y 2017. Se evaluó un total de dos tratamientos contra araña roja en 2016 y 3 tratamientos en 2017.

Las variables respuesta fueron: 1) la distribución del caldo en la copa del árbol (porcentaje de recubrimiento) y 2) la eficacia de control de la plaga (porcentaje de hojas sintomáticas ocupadas por araña roja a los 7, 14 y 21 días del tratamiento).

\subsection{Caracterización de la vegetación}

$\mathrm{Al}$ inicio de la temporada de tratamientos de cada campaña, después de la poda, se estimó el tamaño de la vegetación de la parcela. Para ello, se eligieron al azar 10 árboles representativos y se midió la altura de copa, y el diámetro del árbol a lo largo de la fila y a través de la fila (Figura 1). Con estos datos se calculó el volumen de vegetación considerando la copa del árbol como un elipsoide (Tabla 1).

Tabla 1. Tamaño de los árboles de la parcela.

\begin{tabular}{ccc}
\hline Año & $\begin{array}{c}\text { Dimensiones de la } \\
\text { copa* }^{*} \text { (m) }\end{array}$ & $\begin{array}{c}\text { Volumen de } \\
\text { copa }^{* *}(\mathbf{m} 3)\end{array}$ \\
\hline 2016 & $2,51 \times 4,33 \times 3,08$ & 17,53 \\
2017 & $2,60 \times 4,03 \times 3,05$ & 16,73 \\
\hline
\end{tabular}

* Altura $\times$ diámetro a través de la fila $\times$ diámetro a lo largo de la fila

** Considerando la copa como un elipsoide 

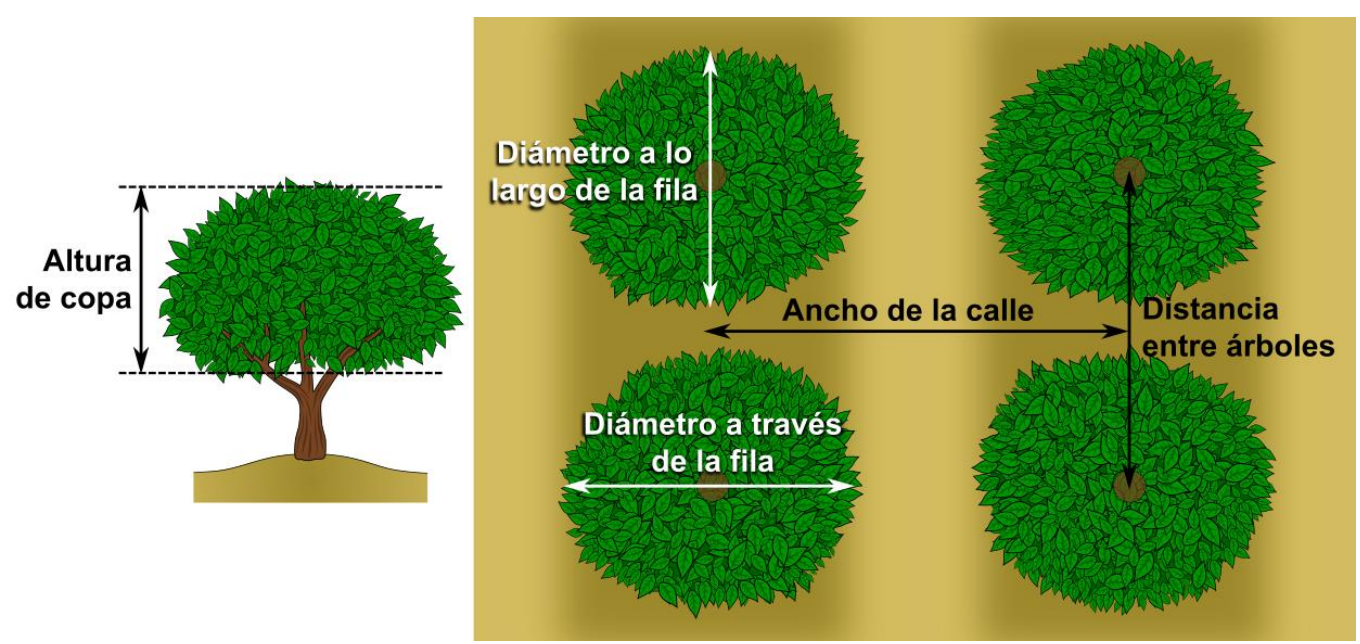

Figura 1. Medidas de marco de plantación y tamaño de copa.

\subsection{Descripción de los tratamientos}

Las aplicaciones se realizaron con un pulverizador hidráulico asistido por aire (mod. IlemoHardi Arrow XF 90. Ilemo Hardi S.A.U., Lleida, España) de 15001 de capacidad y 38 boquillas, arrastrado por un tractor Landini REX 110 F (Landini Argo Tractors S.p.A., Fabbrico, Italia) de 102 $\mathrm{CV}$. Al inicio de cada temporada de tratamientos el equipo se calibró.

VA se basó en la recomendación de CitrusVol, seleccionando los siguientes parámetros:

- Densidad foliar: Media.

- Poda: Normal.

- Plaga/enfermedad: Tetraníquidos (araña roja, ácaro rojo...).

- Productos: Abamectina.

Con esto y las medidas de marco de plantación y tamaño de los árboles, el volumen recomendado por la calculadora ( $\mathrm{V}_{\mathrm{A}}$ ) fue de 3270 l/ha en 2016 y de 3120 1/ha en 2017, lo que implicó una reducción del 29-36 \% respecto a Vc (Tabla 2).

Tabla 2. Volumen de caldo y productos fitosanitarios usados en los tratamientos.

\begin{tabular}{|c|c|c|c|c|c|c|}
\hline \multirow{2}{*}{ Fecha } & \multicolumn{2}{|c|}{$\begin{array}{l}\text { Volumen de } \\
\text { caldo (1/ha) }\end{array}$} & \multirow{2}{*}{$\begin{array}{c}\text { Reducción } \\
\text { de } \\
\text { volumen } \\
\text { de caldo } \\
(\%)\end{array}$} & \multirow{2}{*}{$\begin{array}{c}\text { Producto } \\
\text { fitosanitario }\end{array}$} & \multirow{2}{*}{ Materia activa } & \multirow{2}{*}{$\begin{array}{c}\text { Concentración } \\
\text { de producto } \\
\text { fitosanitario } \\
(\%)\end{array}$} \\
\hline & VC & VA & & & & \\
\hline \multirow{3}{*}{$27 / 07 / 2016$} & \multirow{3}{*}{4905,07} & \multirow{3}{*}{3254,93} & \multirow{3}{*}{33,64} & \multirow{3}{*}{$\begin{array}{c}\text { Cal-Ex } \\
\text { Movento } 150 \\
\text { O-Teq } \\
\text { Envidor }\end{array}$} & Abamectina & 0,100 \\
\hline & & & & & Espirotetramato & 0,040 \\
\hline & & & & & Espirodiclofeno & 0,023 \\
\hline $11 / 10 / 2016$ & 4905,07 & 3254,93 & 33,64 & Cal-Ex & Abamectina & 0,100 \\
\hline \multirow{2}{*}{ 07/06/2017 } & \multirow{2}{*}{4899,00} & \multirow[b]{2}{*}{3487,20} & \multirow[b]{2}{*}{28,82} & Dursban 48 & Clorpirifos & 0,267 \\
\hline & & & & Stygma & Abamectina & 0,100 \\
\hline \multirow{2}{*}{ 24/07/2017 } & \multirow{2}{*}{4899,00} & \multirow{2}{*}{3152,91} & \multirow{2}{*}{35,64} & Abasi EC & Abamectina & 0,100 \\
\hline & & & & Envidor & Espirodiclofeno & 0,023 \\
\hline \multirow{3}{*}{ 08/09/2017 } & \multirow{3}{*}{4899,00} & \multirow{3}{*}{3152,91} & \multirow{3}{*}{35,64} & Reldan E & $\begin{array}{c}\text { Metil- } \\
\text { clorpirifos }\end{array}$ & 0,400 \\
\hline & & & & Dauparex & Abamectina & 0,100 \\
\hline & & & & Envidor & Espirodiclofeno & 0,023 \\
\hline
\end{tabular}

VC: Volumen convencional; VA: Volumen ajustado. 


\section{CONGRESO IBÉRICO DE AGROINGENIERÍA \\ X CONGRESSO IBÉRICO DE AGROENGENHARIA \\ 3 - 6 septiembre 2019, Huesca - España}

Las condiciones operativas se mantuvieron iguales en los tratamientos con $\mathrm{V}_{\mathrm{A}}$ y con $\mathrm{V}_{\mathrm{c}} \mathrm{y}$ fueron: presión de trabajo a 8 bar, velocidad de avance a $1,32 \mathrm{~km} / \mathrm{h}$, régimen de motor y de toma de fuerza de 1800 y $490 \mathrm{rpm}$, respectivamente, y marcha del ventilador II (caudal de aire de $55342,15 \mathrm{~m}^{3} / \mathrm{h}$ en 2016 y $74894,13 \mathrm{~m}^{3} / \mathrm{h}$ en 2017).

Para reducir el volumen de caldo en los tratamientos con $\mathrm{V}_{\mathrm{A}}$ se utilizaron boquillas de menor caudal y se cerraron las boquillas necesarias para ajustar la nube de pulverización a la vegetación con el fin de reducir la deriva y las pérdidas al suelo. Así, los tratamientos con Vc se aplicaron con las 38 boquillas abiertas, mientras que los tratamientos con $\mathrm{V}_{\mathrm{A}}$ se aplicaron con 30 boquillas. La orientación de las boquillas fue la misma para ambos tratamientos.

Para determinar el momento óptimo del tratamiento se realizaron muestreos semanales de seguimiento de las poblaciones de araña roja entre junio y septiembre, siguiendo el protocolo de la guía de Gestión Integrada de Plagas de Cítricos [7].

\subsection{Distribución del caldo en la copa del árbol}

La distribución del caldo en la copa se evaluó mediante el porcentaje de recubrimiento obtenido en diferentes partes de la vegetación. Esta evaluación se realizó en una aplicación de cada campaña, el 27 de mayo de 2016 y el 7 de junio de 2017.

Para ello, previo a la pulverización, se situaron 72 papeles hidrosensibles de $76 \times 26 \mathrm{~mm}$ (Teejet Spraying Systems Co. Wheaton, Illinois, EEUU) por árbol, en 3 árboles por bloque. Los papeles se distribuyeron en 18 zonas de la copa, resultado de dividirla en 3 alturas (alto, medio y bajo), 2 profundidades (interior y exterior) y 3 anchuras (Figura 2). En cada zona se situaron aleatoriamente dos papeles hidrosensibles en el envés de las hojas y dos en el haz.
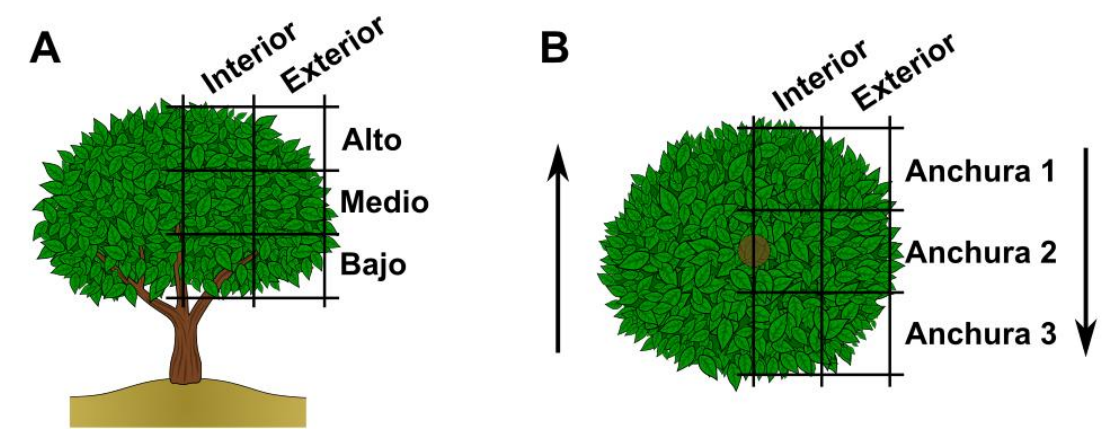

Figura 2. Cuadrantes de la copa. A) vista lateral y B) vista superior de un árbol. Las flechas indican el sentido de avance del turboatomizador respecto a las zonas evaluadas.

Una vez realizada la aplicación, se dejó pasar un tiempo para que se secaran los papeles hidrosensibles, y se recogieron. En laboratorio, los papeles hidrosensibles se digitalizaron con una cámara fotográfica Canon EOS 700D (Canon Inc., Tokyo, Japón) bajo unas condiciones de luz fijas y se analizaron mediante el software de análisis de imagen Food-Color Inspector [8], determinando el porcentaje de recubrimiento.

\subsection{Evaluación de la eficacia de control de araña roja}

Para determinar la eficacia de los tratamientos se realizaron muestreos previos y muestreos posteriores al tratamiento (a los 7, 14 y 21 días), en ambos bloques de la parcela. Para ello, se inspeccionaron 2 hojas sintomáticas por árbol, una del exterior de la copa y otra del interior, en 40 árboles distribuidos al azar en cada bloque, en 20 de ellos se muestrearon hojas de la cara este y en 20 de la cara oeste, y se determinó el porcentaje de hojas sintomáticas ocupadas ( $\geq 2$ arañas rojas adultas). Para el muestreo se descartaron aquellos árboles del contorno de la parcela y de las filas de separación entre bloques de tratamiento. 


\section{CONGRESO IBÉRICO DE AGROINGENIERÍA \\ X CONGRESSO IBÉRICO DE AGROENGENHARIA \\ 3 - 6 septiembre 2019, Huesca - España}

\subsection{Análisis de datos}

En cada momento de aplicación evaluado, las diferencias entre volúmenes en el recubrimiento en cada zona de la copa (combinación de altura $\times$ profundidad $\times$ cara de la hoja) se analizaron mediante un análisis de la varianza (ANOVA) simple. Se comprobó que se cumpliesen los supuestos de homocedasticidad (test de Levene) y de normalidad.

El porcentaje de hojas sintomáticas ocupadas por araña roja con cada volumen aplicado, en cada muestreo realizado, se comparó con un test de chi-cuadrado.

\section{Resultados y discusión}

\subsection{Recubrimiento}

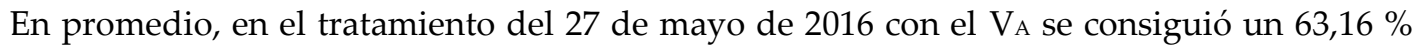
de recubrimiento y con el $V_{c}$ un 75,18 \%, y en el tratamiento del 7 de junio de 2017 un 47,53\% con el $V_{A}$ y un 63,18 \% con el Vc. Promediando ambos tratamientos, se recubrió un 55,10 \% con el $V_{\text {A }}$ y un $69,26 \%$ con el Vc. En la mayoría de las zonas de la copa, se consiguieron porcentajes de recubrimiento más altos con el $\mathrm{V}_{\mathrm{C}}$ que con el $\mathrm{V}_{\mathrm{A}}$, aunque las diferencias sólo fueron significativas en la zona Bajo-Exterior-Envés $(\mathrm{F}=7,77$; g.l. $=1,6 ; \mathrm{P}=0,0494)$ del tratamiento del 27 de mayo de 2016, y en las zonas Alto-Exterior-Envés ( $\mathrm{F}=11,44$; g.l. $=1,6$; $\mathrm{P}=0,0277)$ y BajoInterior-Haz ( $\mathrm{F}=10,39 ;$ g.l. = 1, 6; $\mathrm{P}=$ 0,0322) del tratamiento del 7 de junio de 2017 (Figura 3).

En general, en el exterior de la copa el recubrimiento fue más alto que en el interior y la zona media y baja de la copa de los árboles se mojó más que la parte alta, para ambos volúmenes de aplicación.

\section{7/05/2016}

$\mathbf{V}_{\mathbf{A}}: 3255 \mathrm{l} / \mathrm{ha}\left(0,331 / \mathrm{m}^{3}{ }_{\mathrm{veg}}\right)$ $\mathbf{V}_{\mathbf{C}}: 49051 / \mathrm{ha}\left(0,501 / \mathrm{m}^{3}{ }^{\mathrm{veg}}\right)$

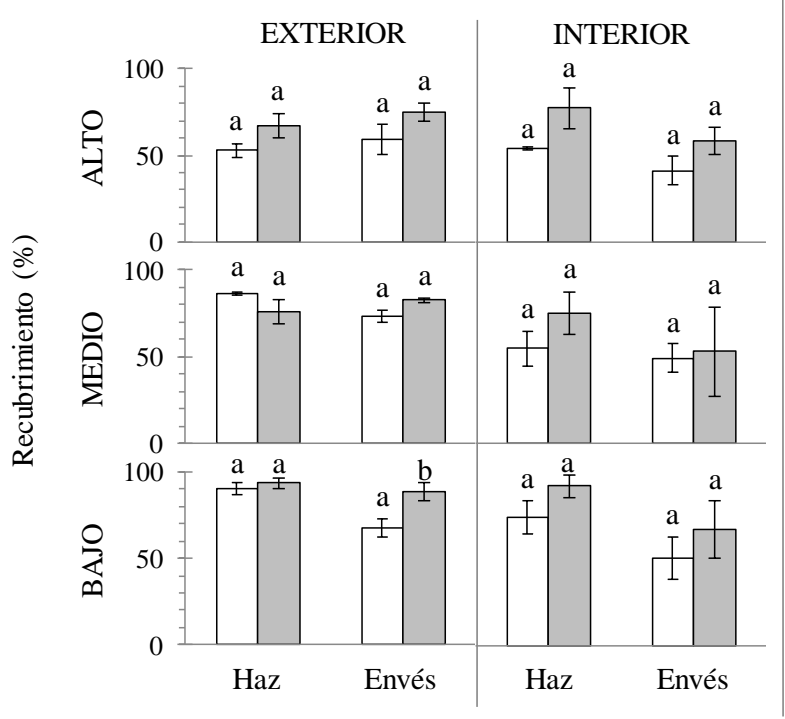

07/06/2017

$\mathbf{V}_{\mathbf{A}}: 3487 \mathrm{l} / \mathrm{ha}\left(0,381 / \mathrm{m}^{3}{ }_{\mathrm{veg}}\right)$ $\mathbf{V}_{\mathbf{C}}: 4899 \mathrm{l} / \mathrm{ha}\left(0,53 \mathrm{l} / \mathrm{m}^{3}{ }^{\mathrm{veg}}\right)$
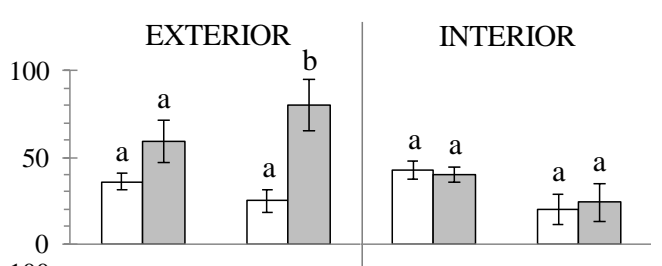

100 - a a a
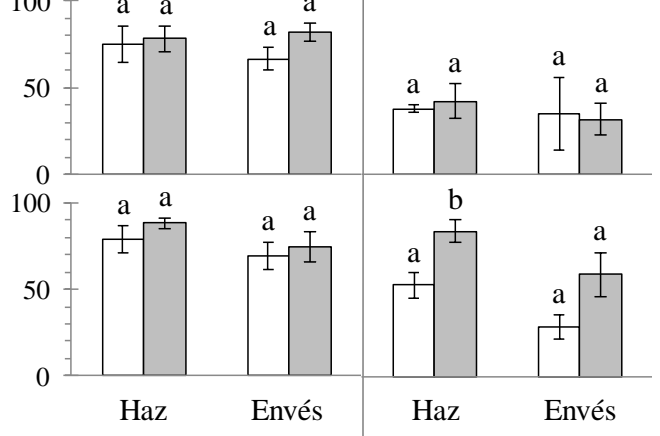

$$
\square \mathrm{v}_{\mathrm{A}} \quad \square \mathrm{v}_{\mathrm{C}}
$$

Figura 3. Recubrimiento (\%; media \pm error estándar) con volumen ajustado $\left(\mathrm{V}_{\mathrm{A}}\right)$ y volumen convencional (Vc) a diferentes alturas (alto, medio y bajo), profundidades (interior y exterior) de la copa y caras de la hoja (haz y envés). Se indica el volumen de caldo en $1 / \mathrm{ha} \mathrm{y} 1 / \mathrm{m}^{3}$ vegetación. Letras diferentes sobre las barras dentro de cada combinación de altura $\times$ profundidad $\times$ cara de la hoja indican diferencias significativas $(\mathrm{P}<0,05)$. 


\section{CONGRESO IBÉRICO DE AGROINGENIERÍA \\ X CONGRESSO IBÉRICO DE AGROENGENHARIA \\ 3 - 6 septiembre 2019, Huesca - España}

\subsection{Porcentaje de hojas sintomáticas ocupadas por araña roja}

Se observó que, independientemente del volumen aplicado, en el interior de la copa hubo más porcentaje de hojas sintomáticas ocupadas que en el exterior. En cuanto a la evolución temporal de la infestación se observó que a los 7 días del tratamiento el nivel de araña roja se redujo, mientras que a los 14 días comenzó a subir. Sin embargo, a los 21 días todavía continuaba en un nivel bajo. Destaca que no hubo diferencias significativas en el control de araña debidas a las diferencias de volumen aplicado (Figura 4).

2016

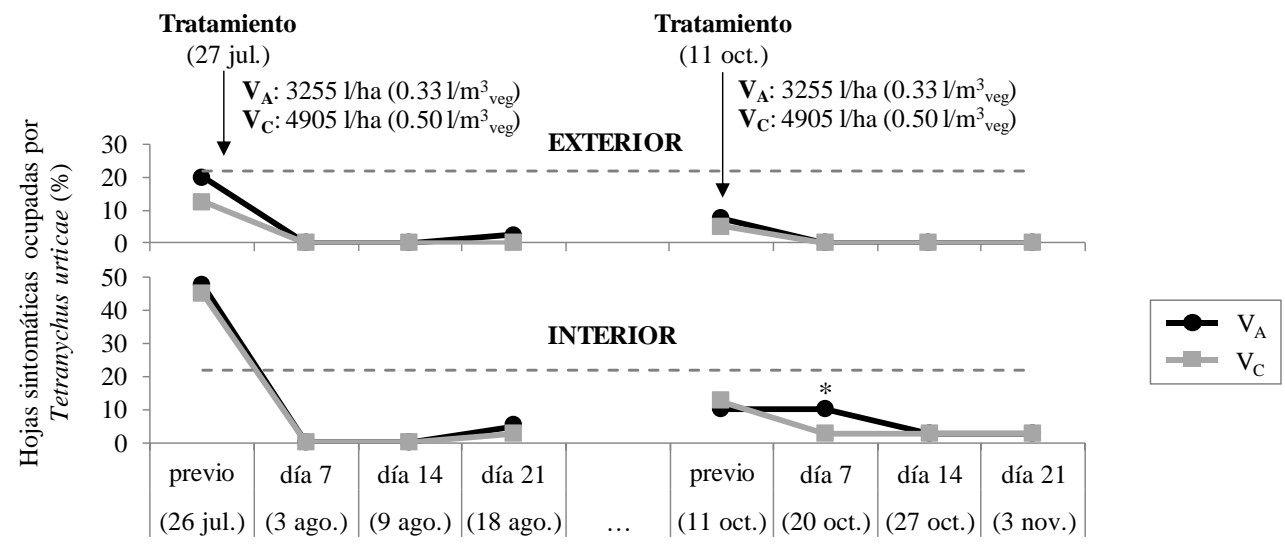

2017

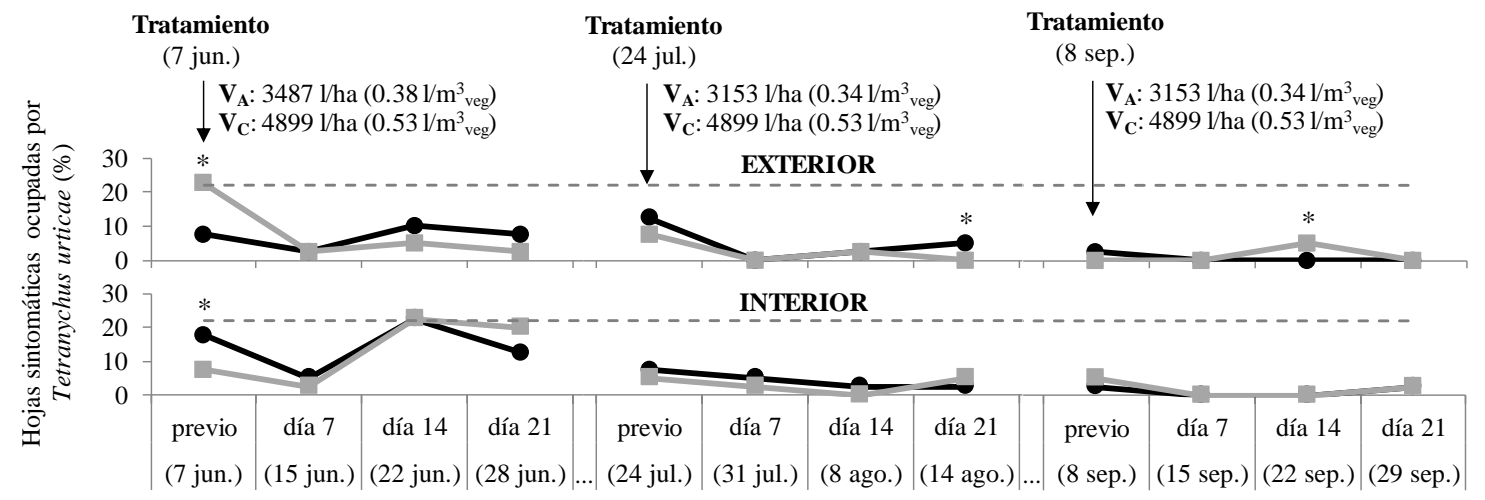

Figura 4. Hojas sintomáticas ocupadas por Tetranychus urticae (\%; promedio), según la situación de la hoja en la copa del árbol (exterior e interior), previamente y después de los tratamientos (a $\operatorname{los} 7,14$ y 21 días) con volumen ajustado $\left(\mathrm{V}_{\mathrm{A}}\right)$ y volumen convencional $\left(\mathrm{V}_{\mathrm{C}}\right)$. Se indica el volumen de caldo en $1 /$ ha y $1 / \mathrm{m}^{3}$ vegetación. La línea de puntos indica el umbral de tratamiento: $22 \%$ de hojas sintomáticas ocupadas. El asterisco $\left({ }^{*}\right)$ indica diferencias significativas con el test de chi-cuadrado (nivel de significación: $\alpha=0,05$ ).

\section{Conclusiones}

Los resultados mostraron que con el empleo de CitrusVol se consiguió una reducción media del volumen de caldo de $33,5 \%$, lo que supuso una reducción del recubrimiento de la copa $(69,3 \%$ frente a 55,1\%). La herramienta recomendó un volumen inferior al empleado por el productor debido a que se ajusta al tamaño y densidad real de la vegetación objetivo, adecuando el volumen de aplicación a la cantidad de vegetación que se pretende proteger. Sin embargo, no se encontraron diferencias significativas en el porcentaje de hojas sintomáticas ocupadas por araña roja en función del volumen de caldo aplicado, lo que indica que el volumen recomendado por la herramienta consiguió un recubrimiento suficiente para controlar la plaga, y que el incremento conseguido con el tratamiento aplicado de forma convencional es 


\section{CONGRESO IBÉRICO DE AGROINGENIERÍA \\ X CONGRESSO IBÉRICO DE AGROENGENHARIA \\ 3 - 6 septiembre 2019, Huesca - España}

innecesario y excesivo. Esto permite concluir que CitrusVol es una herramienta adecuada para la recomendación de volúmenes en el control de Tetranychus urticae en clementinos en las condiciones ensayadas.

\section{Agradecimientos}

Agradecemos a Revacitrus S. L. la cesión de la parcela experimental y su colaboración. Este trabajo ha sido financiado por el IVIA y el instrumento financiero LIFE de la Unión Europea a través del proyecto LIFE PERFECT (LIFE17/ENV/ES/000205).

\section{Referencias}

1. Garcerá C., Moltó E. \& Chueca P. Spray pesticide applications in Mediterranean citrus orchards: canopy deposition and off-target losses. Science of the Total Environment 2017, 599-600, 1344-1362.

2. Garcerá C., Fonte A., Moltó E. \& Chueca P. Sustainable use of pesticide applications in citrus: a support tool for volume rate adjustment. International Journal of Environmental Research and Public Health. 2017, 14(7), 715. doi: 10.3390/ijerph14070715.

3. Garcerá C. Racionalización de las aplicaciones de productos fitosanitarios para el control de Aonidiella aurantii Maskell (Hemiptera: Diaspididae) en cítricos. Tesis doctoral, Universitat Politècnica de València, València, España, Julio 2013.

4. Garcerá C., Moltó E. \& Chueca P. Factors influencing the efficacy of two organophosphate insecticides in controlling California red scale, Aonidiella aurantii (Maskell). A basis for reducing spray application volume in Mediterranean conditions. Pest Management Science. 2014, 70, $28-38$.

5. Garcerá C., Moltó E. \& Chueca P. Effect of spray volume of two organophosphate pesticides on coverage and mortality of Aonidiella aurantii Maskell. Crop Protection. 2011, 30, 693-697.

6. Garcerá C., Moltó E., Zarzo M. \& Chueca P. Modelling the spray deposition and efficacy of two mineral oil-based products for the control of California red scale, Aonidiella aurantii (Maskell). Crop Protection. 2012, 31, 78-84.

7. Ministerio de Agricultura, Alimentación y Medio Ambiente. Gestión Integrada de Plagas de Cítricos. Centro de Publicaciones del MAGRAMA, Madrid, España. 2014. ISBN: 978-84-491-1411-3. 159 pp.

8. Albert F., Aleixos N., Blasco J., \& Cubero, S. Food Color Inspector. 2013. Accesible desde http://www.cofilab.com/registered-software/ [10/05/2019]. 